\title{
Options can induce risk taking for arbitrary preferences ${ }^{\star}$
}

\author{
Luis H.B. Braido ${ }^{1}$ and Daniel Ferreira ${ }^{2}$ \\ 1 Graduate School of Economics, Getulio Vargas Foundation, Praia de Botafogo 190, s.1100, \\ Rio de Janeiro, RJ 22253-900, BRAZIL (e-mail: lbraido@fgv.br) \\ 2 SITE, Stockholm School of Economics, Box 6501, SE 11383 Stockholm, SWEDEN \\ (e-mail: Daniel.Ferreira@hhs.se)
}

Received: November 5, 2003; revised version: November 1, 2004

Summary. It is widely believed that call options induce risk-taking behavior. However, Ross (2004) challenges this intuition by demonstrating the impossibility of inducing managers with arbitrary preferences to always act as if they were less risk averse. If preferences and price distributions are unknown, risk-taking behavior cannot be always induced by an option contract. Here, we prove a new result showing that, with no information about preferences and some knowledge about prices, one can write a call option that makes all managers prefer riskier projects to safer ones. This points out that in order to design options that induce risk taking it is sufficient to have information about price distributions.

Keywords and Phrases: Stochastic dominance, Stock options, Risk taking.

JEL Classification Numbers: D81, G00, J33, M21.

\section{Introduction}

Regardless of the reasons why firms grant stock options to their executives, it is widely believed that a consequence of this practice is an increase in managerial willingness to take risks. Intuitively, it is reasonable to expect that compensation schedules based on call options would induce risk taking, since call options display convex payoff structures and their values increase with the volatility of the underlying stock - see Smith and Stulz (1985) and DeFusco, Johnson, and Zorn (1990). However, as noticed by Ross (2004), this argument ignores the fact that

\footnotetext{
* We thank Renée Adams, Heitor Almeida, Carlos E. da Costa, Andrew Horowitz, Paulo K. Monteiro, Walter Novaes, Sergio O. Parreiras, Rodrigo R. Soares, and especially Marcos Tsuchida for many helpful comments.
} 
option-based compensation schemes move the evaluation of a given lottery to a locus in the domain where utility functions might be more concave. Thus, the convex shape of call payoffs could be offset by locally higher degrees of risk aversion. ${ }^{1}$ In fact, Ross (2004) proves an impossibility theorem. He shows that, among the class of strictly convex compensation schedules, there is no single schedule that would make all strictly concave utility functions uniformly display lower risk aversion everywhere in their domains. Based on this result, Ross (2004) states that "the common folklore that giving options to agents will make them more willing to take risks is false."

In this paper, we ask what the conditions are under which call options may induce risk taking. We prove a new result that is helpful in understanding the relationship between options and risk taking. If $F$ and $G$ are two cumulative distribution functions and $F$ second-order stochastically dominates $G$ (i.e. $G$ is unambiguously riskier than $F$ ), then there is a censoring point such that the distribution $G$ censored at this point first-order stochastically dominates the distribution $F$ censored at the same point.

Because call options pay only if the stock price surpasses a threshold level (the strike price), the payoff distribution implied by a call option is a linear transformation of the original stock-price distribution censored at the strike price. According to our result, by choosing a proper censoring point (i.e., an appropriate strike price), one can transform a project that is unambiguously riskier than others into a lottery that first-order stochastically dominates all others. Consequently, executives facing compensation schedules based on call options with such strike prices will always prefer to implement riskier projects, regardless of the specific functional form of their utility functions. We also show that this result is robust to scenarios in which one has limited information about the price probability distributions implied by each project.

These findings are complementary to Ross's (2004) in the following sense. Given Ross's impossibility result, one needs to further restrict the environment in order to get some possibilities. By restricting the class of admissible utility functions, Ross (2004) is able to find convex compensation schedules that would make any agent displaying decreasing absolute risk aversion become less risk averse. Our possibility theorem takes an alternative route. We do not restrict the class of utility functions; in fact, utilities here can be concave, convex, or neither. We show that if the distribution functions of the available projects are at least partially known, then one can find call options that would make all agents behave as if they were less risk averse, regardless of their risk preferences. Thus, this paper identifies the information about projects' distributions as a key determinant of the risk-taking inducing property of call options.

Two important points are worth stressing. First, as in Ross (2004), we do not adopt an optimal-contracting approach. It is not our goal to explain why options are used in executive compensation. Instead, we focus on a more basic question: Can stock options be used to make managers choose riskier projects over safer ones regardless of their risk preferences? Second, the novelty of our result is not

${ }^{1}$ Qualitatively similar results are derived by Carpenter (2000) and Lewellen (2002). 
that using option contracts can induce agents to prefer riskier projects. This result is obviously true for some price distributions and utility functions. The not-so-obvious result proven here is that it is possible to find one option contract that would make all agents behave as if they were less risk averse, regardless of their utility functions. In practice, option contracts are very homogeneous across managers, thus it is important to find out which properties of option contracts are robust to arbitrary preferences.

Our exposition is organized in the following manner. We present our setup and main results in Section 2. In Section 3, we apply the analysis to two commonly used families of distribution functions: Lognormal and Normal. Interestingly, the rules for determining robust strike prices are extremely simple. Section 4 serves as a brief conclusion.

\section{Call options and risk taking}

Consider a firm run by a manager whose actions have stochastic effects on the firm's stock price $(t)$. The manager has to implement only one project among those that are available. Contracts specifying which project the manager must choose are not enforceable. Managers have no private preferences over projects. The economy lasts for one period and different projects induce different stock-price distributions for the next period. Managers' preferences have the expected-utility property and their utility functions over wealth, $u: \mathbb{R}_{+} \rightarrow \mathbb{R}$, are continuous and increasing.

Our risk metric for now will be the concept of second-order stochastic dominance. For any two distribution functions $F:[0, T] \rightarrow[0,1]$ and $G:[0, T] \rightarrow[0,1]$ such that $F \neq G$, one has that $F$ second-order stochastically dominates $G\left(F \succsim_{s} G\right)$ if:

$$
\begin{gathered}
E_{F}(t)=E_{G}(t), \text { and } \\
\int_{0}^{\tau} G(t) d t \geq \int_{0}^{\tau} F(t) d t, \forall \tau \in[0, T] .
\end{gathered}
$$

Our goal is to find a compensation schedule that would induce any type of manager to implement the riskiest project available, regardless of the specific functional form of $u$. When these schedules exist, we say that they are robust to different preferences.

We restrict our analysis to one of the simplest convex schedules used in managerial compensation: call options of stock prices. In our setup, an option is fully specified by its strike price, $s$. When a manager with utility $u$ is faced with a call option with strike price $s$, his expected utility is given by:

$$
U(s, H)=\int_{0}^{T} u(\max (t-s, 0)) d H(t),
$$

where $H$ represents a generic distribution function for $t$. 


\subsection{Two-project environment}

Initially, we consider the case in which there are two projects available and extend the analysis later. Fix $F$ and $G$ to be such that $F \neq G$ and $F \succsim_{s} G$ in order to compute risk-taking inducing strike prices that might depend on $F$ and $G$, but are independent of managers' utilities.

Before stating our main result, we need a technical assumption to rule out distribution functions displaying pathological behaviors in a neighborhood of the stock-price upper bound $(T)$. Define $T_{F G}$ as the limit point at which the two distributions differ from each other, i.e.:

$$
T_{F G}=\min \{\theta \in[0, T]: F(t)=G(t) \text { for all } t \in[\theta, T]\} .
$$

Notice that $T_{F G}$ always exists, since $F(T)=G(T)=1$ and distribution functions are right continuous. (Also, $T_{F G}=T$ unless $F(t)=G(t)$ in a neighborhood of $T$.) We thus make the following assumption.

Assumption 1. There is a neighborhood of $T_{F G}$ in which the number of times $F(t)-G(t)$ changes sign is finite.

Assumption 1 is very weak. In particular, it is satisfied by any pair of probabilities with finite support and all standard distribution functions such as Normal, Lognormal, Chi-Square, Gama, Beta, Weibull, t and F, among others. We are now ready to state our main result.

Proposition 1. Under Assumption 1, there exists a strike price $\hat{s} \in(0, T)$ such that:

$$
\int_{0}^{T} u(\max (t-\hat{s}, 0)) d G(t)>\int_{0}^{T} u(\max (t-\hat{s}, 0)) d F(t),
$$

for any continuous and increasing $u$. In other words, there always exists a call option that is robust to preferences and induces any type of manager to choose the riskier project.

Proof. Under Assumption 1, if $F \succeq_{s} G$ then $\exists \delta>0$ such that $G(t)<F(t), \forall t \in$ $\left(T_{F G}-\delta, T_{F G}\right)$. To see this, first note that Assumption 1 implies that there exists $\delta>0$ such that $F(t)-G(t)$ is either positive or negative, $\forall t \in\left(T_{F G}-\delta, T_{F G}\right)$. Moreover, from the definition of second-order stochastic dominance, one must have $\int_{\tau}^{T_{F G}}(G(t)-F(t)) d t \leq 0, \forall \tau<T_{F G} .^{2}$ Hence, $G(t)-F(t)$ must be negative, $\forall t \in\left(T_{F G}-\delta, T_{F G}\right)$.

Next, define $A=\{t \in[0, T]: G(t)>F(t)\}$. The set $A$ is non-empty, otherwise we would have either $F=G$ or $E_{F}(t)>E_{G}(t)$. Moreover, it must be the case that $\sup (A)<T_{F G}$, since $\exists \delta>0$ such that $G(t)<F(t), \forall t \in$ $\left(T_{F G}-\delta, T_{F G}\right)$. Define then $\hat{s}=\sup (A)$ and let $G^{o}:[0, T-\hat{s}] \rightarrow[0,1]$ and $F^{o}:[0, T-\hat{s}] \rightarrow[0,1]$ denote the distributions of $\max (t-\hat{s}, 0)$ when $t$ follows $F$ and $G$, respectively. Notice that the definition of $\hat{s}$ and right continuity of $F$ and

\footnotetext{
2 Notice that, $E_{F}(t)-E_{G}(t)=0 \Leftrightarrow \int_{0}^{T} t(d G(t)-d F(t))=0 \Leftrightarrow-\int_{0}^{T}(G(t)-d F(t)) d t=0 \Leftrightarrow$ $\int_{0}^{T_{F G}} G(t) d t=\int_{0}^{T_{F G}} F(t) d t$. The inequality follows thus from equation (2).
} 


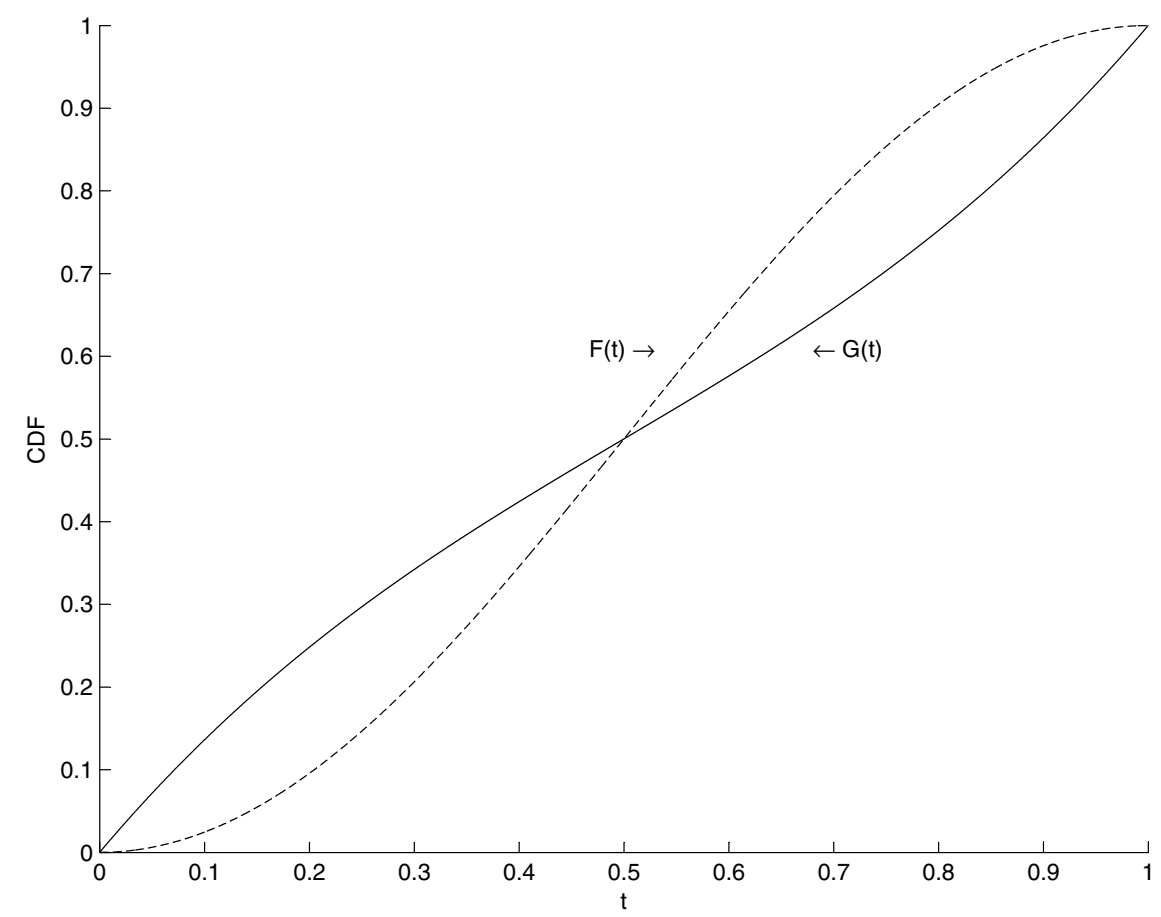

Figure 1

$G$ imply $G^{o}(t)=G(\hat{s}+t) \leq F^{o}(t)=F(\hat{s}+t), \forall t \in[0, T-\hat{s}]$. Therefore, $G^{o}$ first-order stochastically dominates $F^{o}$.

Finally, notice that $\exists \delta>0$ such that $\hat{s}=\sup (A) \leq T_{F G}-\delta$ and $G^{o}(t)=$ $G(\hat{s}+t)<F(\hat{s}+t)=F^{o}(t), \forall t \in\left(T_{F G}-\delta-\hat{s}, T_{F G}-\hat{s}\right)$. Therefore, one has $\int_{0}^{T} u(\max (t-\hat{s}, 0)) d G(t)=\int_{0}^{T-\hat{s}} u(t) d G^{o}(t)>\int_{0}^{T-\hat{s}} u(t) d F^{o}(t)=$ $\int_{0}^{T} u(\max (t-\hat{s}, 0)) d F(t)$, for any continuous and increasing $u$.

Our proof is constructive, thus one can compute the strike price that induces such a result, namely $\hat{s}=\sup \{t \in[0, T]: G(t)>F(t)\}$. Figure 1 displays two cumulative distribution functions to illustrate our proof. In this figure, $T_{F G}=T=$ 1 and $\hat{s}=0.5$. Notice that, under Assumption $1, F \succeq_{s} G$ implies $G(t)<F(t)$ for all $t \in\left(\hat{s}, T_{F G}\right)$. But this implies that $G$ always yields higher payoffs than $F$ when the domain is restricted to values higher than $\hat{s}$, implying that any agent that prefers more money to less should prefer $G$. In other words, a call option that censors both distributions at point $\hat{s}$ would generate two new distributions, $F^{o}$ and $G^{o}$, such that $G^{o}$ first-order stochastically dominates $F^{o}$.

Proposition 1 is considered our main result because all the other results that follow can be seen as extensions or variations of it. The next proposition, for instance, generalizes the previous result.

Proposition 2. Define $\hat{s}=\sup \{t \in[0, T]: G(t)>F(t)\}$ and suppose Assumption 1 holds. Then: 
(i) For any $s^{\prime} \in\left[\hat{s}, T_{F G}\right)$, one has:

$$
\int_{0}^{T} u\left(\max \left(t-s^{\prime}, 0\right)\right) d G(t)>\int_{0}^{T} u\left(\max \left(t-s^{\prime}, 0\right)\right) d F(t),
$$

for any continuous and increasing $u$.

(ii) If $s^{\prime} \geq T_{F G}$, the inequality sign in (6) should be replaced by an equality sign.

Proof. Similarly to the proof of Proposition 1, define $G^{o}(t)=G\left(s^{\prime}+t\right)$ and $F^{o}(t)=F\left(s^{\prime}+t\right)$, where $s^{\prime} \geq \hat{s}$. From the definition of $\hat{s}$, one must have $G^{o} \leq F^{o}$ (which implies that $G^{o}$ first-order stochastically dominates $F^{o}$ ). If $s^{\prime} \in\left[\hat{s}, T_{F G}\right.$ ), one must have $G^{o}<F^{o}$ for some open interval in $\left[\hat{s}, T_{F G}\right)$, which implies the strict inequality in (6). Moreover, $G^{o}=F^{o}$ if $s^{\prime} \geq T_{F G}$, which implies part (ii).

This result is important because it suggests that fine-tuning the strike price is not necessary in order to induce the risk-taking behavior. With limited information about the environment (i.e., with imperfect estimates of $F$ and $G$ ), one may choose to overshoot the strike price to guarantee that $G$ is chosen over $F$. Ideally, one should choose $s^{\prime} \in\left[\hat{s}, T_{F G}\right)$, but if by mistake $s^{\prime}$ is set to be larger than $T_{F G}$, managers would still be indifferent between the projects. Therefore, we have shown that with no information about preferences and with only rough estimates of $F$ and $G$, one can find a family of option-based compensation schedules which induce the choice of risky projects. In this sense, these schedules are not only robust to preferences, but they are also robust to some small perturbations of the functions $F$ and $G$.

\subsection{Multiple projects}

We now study the case where managers choose among many different projects. The basic intuition remains the same, but we need some additional assumptions.

The concept of second-order stochastic dominance defines a partial ordering of projects, that is: $\succsim_{s}$ is reflexive $\left(F \succsim_{s} F\right.$ ); antisymmetric (if $F \succsim_{s} G$ and $G \succsim_{s} F$ then $F=G$ ); and transitive (if $F \succsim_{s} G$ and $G \succsim_{s} H$ then $F \succsim_{s} H$ ). Thus, define $\Omega$ as a set of distribution functions linearly ordered by $\succsim_{s}$ (where a set is said to be linearly ordered by a partial order $\succsim_{s}$ if, for any two distinct elements $F$ and $G$, one has that either $F \succsim_{s} G$ or $G \succsim_{s} F$ ).

Now, suppose that a manager is exogenously exposed to a finite number of projects and has to decide which one will be implemented. Technically, the manager must implement a project selected from a finite subset of $\Omega$, say $\hat{\Omega}$, which is assumed to be known. Since $\hat{\Omega}$ is finite, there exists the riskiest project, $G^{*} \in \hat{\Omega}$. We introduce Assumption 2, which is analogous to Assumption 1 for the multiproject setup.

Assumption 2. For each $F \in \hat{\Omega}$, there is a neighborhood of $T_{F G^{*}}$ in which the number of times $F(t)-G^{*}(t)$ changes sign is finite.

We can prove two related results in this setup. If $T_{F G^{*}}$ is constant for all $F$ in $\hat{\Omega} \backslash\left\{G^{*}\right\}$, then one can generalize Proposition 1. This technical condition is satisfied, for instance, if $G^{*} \neq F$ almost everywhere, for all $F \in \hat{\Omega} \backslash\left\{G^{*}\right\}$. However, 
when such a condition does not hold, we can still prove a weaker version of Proposition 1 in which the manager weakly (rather than strictly) prefers the option written on the riskiest project. In any case, given a finite set of projects, one can always find a call option that would induce any type of manager to choose the riskiest project available.

Proposition 3. Under Assumption 2, there exists a strike price $s_{\max } \in(0, T)$ such that:

(i) If $T_{F G^{*}}=T^{*}$ for all $F \in \hat{\Omega} \backslash\left\{G^{*}\right\}$, then:

$$
\int_{0}^{T} u\left(\max \left(t-s_{\max }, 0\right)\right) d G^{*}(t)>\int_{0}^{T} u\left(\max \left(t-s_{\max }, 0\right)\right) d F(t),
$$

for any $F \in \hat{\Omega} \backslash\left\{G^{*}\right\}$ and any continuous and increasing $u$;

(ii) If $T_{F G^{*}}$ is not constant for all $F \in \hat{\Omega} \backslash\left\{G^{*}\right\}$, then (7) holds with weak inequality.

Proof. Fix $F \in \hat{\Omega}$ and define $\hat{s}_{F}=\sup \left\{t \in[0, T]: G^{*}(t)>F(t)\right\}$. Since $\hat{\Omega}$ is finite, there is a maximum strike price, say $s_{\max }=\max \left(\hat{s}_{F}, \forall F \in \hat{\Omega} \backslash\left\{G^{*}\right\}\right)$. From Proposition 1, we know that a call option with a strike price equal to $\hat{s}_{F}$ would induce the choice of $G^{*}$ over $F$. If $T_{F G^{*}}=T^{*}$ for all $F \in \hat{\Omega} \backslash\left\{G^{*}\right\}$, then $s_{\max }<T^{*}$ and part (i) is guaranteed by Proposition 2. Otherwise, one might have $s_{\max } \geq T_{F G^{*}}$ for some $F$, making the option written on $F$ identical to the option written on $G^{*}$.

\subsection{Random binary choices}

So far we have assumed that the manager's choice set is known. A different problem arises when one is uncertain about which choices are feasible for the manager.

As in the previous subsection, suppose there is a finite set of projects available, $\hat{\Omega}$, and that these projects are ordered by second-order stochastic dominance. But now the manager is randomly exposed to a pair of them. The set of all available projects is known, but one does not know which specific pair of projects will be available to the manager. We need to find a call option that would induce any type of manager to choose the riskier of any two projects, for any possible pair in $\hat{\Omega} \times \hat{\Omega}$.

Clearly, inducing this type of behavior is much more difficult than inducing the choice of a particular project, as done in the previous subsection. Nevertheless, we have a proposition with the same flavor of the previous ones. We introduce Assumption 3 before stating our result.

Assumption 3. For each pair $(F, G) \in \hat{\Omega} \times \hat{\Omega}$, there is a neighborhood of $T_{F G}$ in which the number of times $F(t)-G(t)$ changes sign is finite.

Proposition 4. Under Assumption 3, there exists a strike price $s_{\max } \in(0, T)$ such that: 
(i) If $T_{F G}=T^{*}$ for all $(F, G) \in \hat{\Omega} \times \hat{\Omega}$, then:

$$
\int_{0}^{T} u\left(\max \left(t-s_{\max }, 0\right)\right) d G(t)>\int_{0}^{T} u\left(\max \left(t-s_{\max }, 0\right)\right) d F(t),
$$

for any $(F, G) \in \hat{\Omega} \times \hat{\Omega}$ such that $F \succsim_{s} G$, and any continuous and increasing $u$;

(ii) If $T_{F G}$ is not constant for all $(F, G) \in \hat{\Omega} \times \hat{\Omega}$, then (8) holds with weak inequality.

Proof. Fix a pair $(F, G) \in \hat{\Omega} \times \hat{\Omega}$ where $F \succeq_{s} G$ (this is without loss of generality, since $\hat{\Omega}$ is linearly ordered by $\succeq_{s}$ ). From Proposition 1 , there is a strike price $\hat{s}_{F G}=\sup \{t \in[0, T]: G(t)>F(t)\}$ that induces any manager to strictly prefer calls written on $G$. Since $\hat{\Omega} \times \hat{\Omega}$ is finite, there is a finite set of strike prices defined in this way. Take the maximum of these strike prices, $s_{\max }=\max \left\{\hat{s}_{F G}, \forall(F, G) \in\right.$ $\hat{\Omega} \times \hat{\Omega}\}$. Note that $s_{\max }<T^{*}$ in case (i) but, in case (ii), one might have $s_{\max } \geq$ $T_{F G}$, for some $(F, G) \in \hat{\Omega} \times \hat{\Omega}$. The result follows then from Proposition 2 .

This proposition illustrates another kind of robustness property of option-based compensation schedules. It shows that wisely chosen schedules can induce risk taking when preferences are unknown, even without complete information about the choices that will be available to the manager.

\section{Simple rules under some standard distributions}

Here, we apply the previous analysis to two commonly used families of distribution functions: Lognormal and Normal. Unlike in the previous sections, we use variance as our measure of risk. This is mainly for simplicity: it facilitates the analysis and allows us to work with unbounded supports. It should be clear that the same propositions we have derived so far could be applied in the current setting, once one truncates the support. Incidentally, the examples of this section show that, for some distribution functions, neither the bounded-support assumption nor the use of second-order stochastic dominance as the risk metric are necessary for robust risk-taking inducing option schedules to exist.

The most interesting results of this section concern the simplicity of the rules used to compute strike prices. Here, the strike price takes the form of a simple function of some moments of the distributions.

\subsection{Lognormal distributions}

Let $F$ and $G$ represent two different projects following Lognormal distributions with the same mean, but different variances. Project $F$ induces a distribution of prices such that $\ln (t) \sim N\left(\mu_{F}, \sigma_{F}^{2}\right)$. Similarly, project $G$ implies $\ln (t) \sim N\left(\mu_{G}, \sigma_{G}^{2}\right)$. Then $F(t)=\Phi\left(\frac{\ln (t)-\mu_{F}}{\sigma_{F}}\right)$ and $G(t)=\Phi\left(\frac{\ln (t)-\mu_{G}}{\sigma_{G}}\right)$, where $\Phi$ represents the standard normal cumulative distribution. We assume that $\sigma_{G}^{2}>\sigma_{F}^{2}$ so that $\operatorname{var}_{G}(t)>\operatorname{var}_{F}(t)$, i.e., $G$ is riskier than $F$. 
Notice that $F$ and $G$ are equal to each other at the points 0 and $\hat{s}$ (and when $t \rightarrow \infty)$, where $\hat{s}$ is given by:

$$
F(\hat{s})=\Phi\left(\frac{\ln (\hat{s})-\mu_{F}}{\sigma_{F}}\right)=\Phi\left(\frac{\ln (\hat{s})-\mu_{G}}{\sigma_{G}}\right)=G(\hat{s}) .
$$

By assumption, $F$ and $G$ have the same mean $(\bar{t})$, where:

$$
\bar{t}=E_{F}(t)=\exp \left(\mu_{F}+\frac{\sigma_{F}^{2}}{2}\right)=\exp \left(\mu_{G}+\frac{\sigma_{G}^{2}}{2}\right)=E_{G}(t) .
$$

Using (10) and (9), one obtains:

$$
\hat{s}=\bar{t} \exp \left(\frac{\sigma_{G} \sigma_{F}}{2}\right) .
$$

Note that if the stock-price mean and variance induced by each project were unknown, one still could use an upper-bound estimate for these variables (say, $\bar{t}_{\max }$ and $\left.\sigma_{\max }^{2}\right)$ and set the strike price to be $s^{\prime}=\bar{t}_{\max } \exp \left(\frac{\sigma_{\max }^{2}}{2}\right)$.

\subsection{Normal distributions}

Let $F(t)=\Phi\left(\frac{t-\mu_{F}}{\sigma_{F}}\right)$ and $G(t)=\Phi\left(\frac{t-\mu_{G}}{\sigma_{G}}\right)$ represent two different projects, where $\Phi$ is the standard Normal cumulative distribution. ${ }^{3}$ These functions are equal to each other at $\hat{s}$ (and when $t \rightarrow \pm \infty$ ), where $\hat{s}$ is the solution of:

$$
F(\hat{s})=\Phi\left(\frac{\hat{s}-\mu_{F}}{\sigma_{F}}\right)=\Phi\left(\frac{\hat{s}-\mu_{G}}{\sigma_{G}}\right)=G(\hat{s}),
$$

that is,

$$
\hat{s}=\mu_{F}=\mu_{G} .
$$

In the case of Normal distributions (or others that can be reasonably approximated by them), the only information needed is the expected stock price. By setting $\hat{s}$ equal to the average stock price, one is able to induce any type of manager to choose the riskier project regardless of $\sigma_{G}$ and $\sigma_{F}$.

\section{Final remarks}

Ross's (2004) results suggest that call options might be a very ineffective way of inducing risk-taking behavior. This is particularly true in cases in which one has limited information about the environment. A firm that would like to force its manager to implement a risky project is typically unaware of the specific functional form of the manager's utility function, and might also have imperfect information about the projects' stochastic properties. In this paper, we studied conditions under which call options may robustly induce risk taking. We showed that it is the lack of information about projects' distributions, rather than the absence of information about preferences, that may hinder the use of call options as a means of inducing managerial risk taking.

\footnotetext{
3 Obviously, price distributions cannot be Normal, since prices must be nonnegative. We use this case only to illustrate our method.
} 


\section{References}

Carpenter, J.N. (2000) Does option compensation increase managerial risk appetite? Journal of Finance 55, 2311-2331

DeFusco, R.A., Johnson, R.R., Zorn, T.S. (1990) The effect of executive stock option plans on stockholders and bondholders. Journal of Finance 45, 617-627

Lewellen, K. (2002) Financing decisions when managers are risk averse. Mimeo, University of Rochester Ross, S.A. (2004) Compensation, incentives, and the duality of risk aversion and riskiness. Journal of Finance 59, 207-225

Smith, C.W., Stulz, R. (1985) The determinants of firms' hedging policies. Journal of Financial and Quantitative Analysis 20, 391-405 